\title{
Improving the range of UHF RFID transponders using solar energy harvesting under low light conditions
}

\author{
A. Ascher, M. Lehner, M. Eberhardt, and E. Biebl \\ Fachgebiet Höchstfrequenztechnik, Technische Universität München, Munich, Germany \\ Correspondence to: A. Ascher (alois.ascher@tum.de)
}

Received: 1 December 2014 - Revised: 14 April 2015 - Accepted: 4 May 2015 - Published: 3 November 2015

\begin{abstract}
The sensitivity of passive UHF RFID transponders (Radio Frequency Identification) is the key issue, which determines the maximum read range of an UHF RFID system. During this work the ability of improving the sensitivity using solar energy harvesting, especially for low light conditions, is shown. To use the additional energy harvested from the examined silicon and organic solar cells, the passive RFID system is changed into a semi-active one. This needs no changes on the reader hardware itself, only the used RFIC (Radio Frequency Integrated Circuit) of the transponder has to possess an additional input pin for an external supply voltage. The silicon and organic cells are evaluated and compared to each other regarding their low light performance. The different cells are examined in a shielded box, which is protected from the environmental lighting. Additionally, a demonstrator is shown, which makes the measurement of the extended read range with respect to the lighting conditions possible. If the cells are completely darkened, the sensitivity gain is ascertained using high capacity super caps. Due to the measurements an enhancement in range up to $70 \%$ could be guaranteed even under low light conditions.
\end{abstract}

\section{Introduction}

RFID denotes a system that allows an automatically detection and localizing process. The communication between an UHF RFID transponder and the corresponding reader comprises of an UHF reader sending a RF signal, which is detected by the transponder. The transponder, which is consisting of an antenna and an RFIC chip, is operated with the received power of the reader. The reader signal is used for supplying the internal logic of the IC and the backscatter to the reader itself. The maximum detection range is limited by law because of sending-power restrictions and the given environmental conditions for a specific case of application. Depending on the environmental requirements the detection range of an RFID system can be improved. Nowadays RFID systems are utilized in different applications, which exhibit various requirements in case of detection range and reliability. For monitoring of issued goods in a warehouse the dominating factor is reliability. The detection range is not that important, because the goods are very close to the reader. In contrast a system for gathering the freeway toll must be both, very reliable and with a large detection range. To guarantee high ranges with RFID systems without using an additional battery a solution using solar energy harvesting is proposed. Based on the concept of a passive UHF RFID system a semi-active localizing system is developed. Thereby no changes on the reader itself are needed. Only the transponder is changed with the goal to get the internal supply of the used RFIC independent of the received reader signal power. For that issue solar energy harvesting should be applied (Spies et al., 2007). Thereby amorphous silicon solar cells from Vimun and Powerfilm, which are commonly used in energy harvesting solutions (Georgiadis and Collado, 2011; Sample et al., 2011), and organic solar cells from the Fraunhofer ISE, which have some advantages in low light performance (Dennler and Sariciftci, 2005), are evaluated and compared to each other. In many cases solar energy harvesting in RFID applications is described using optimal illumination scenarios or no further information about the needed illuminance is given. So the focus shall be onto the low light performance of a possible transponder for indoor applications, using different types of solar cells linked to the maximum read range of the RFID system. Several research activities for performant silicon solar cells with high efficiencies at low light conditions (Glunz et al., 2002) show, 


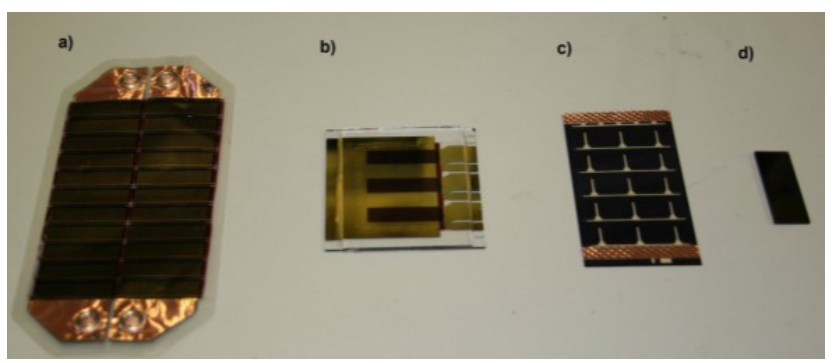

Figure 1. Used solar cells: (a) organic panel; (b) organic single cells; (c) Powerfilm SP 3-37; (d) Vimun Sc-3012.

that the operation of autonomous solar powered devices for indoor applications is possible. The case of completely darkened solar cells should be intercepted in our case and a potential transponder should work with its higher sensitivity for a few hours using an additional capacitor. Finally, based on the previous results, a variable demonstrator is build with the possibility to examine different types of antennas, capacitors and solar cells regarding the illuminance and the achievable reading range.

The following demonstrator-transponder uses the Impinij Monza X-2K RFIC, which has a reading sensitivity of $-17 \mathrm{dBm}$ without- and a reading sensitivity of $-24 \mathrm{dBm}$ with an additional external voltage supply. Therefore, the minimum voltage supply is $1.6 \mathrm{~V}$ at a minimum current of $-17 \mu \mathrm{A}$. Using the demonstrator read sensitivity of $-17 \mathrm{dBm}$ and the Friis equation (Finkenzeller, 2008) the maximum detection range is $11.15 \mathrm{~m}$ with a dipol as transponder antenna and the maximum EIRP sending power of $33 \mathrm{dBm}$ at the reader. The maximum detection range for the same setting, excepting a read sensitivity of $-24 \mathrm{dBm}$, is $24.96 \mathrm{~m}$. The difference in distance is the maximum achievable gain for the detection range limited by the sensitivity of the demonstrator-transponder.

\section{Evaluating solar cells}

For evaluating the amorphous silicon solar cells Powerfilm SP 3-37 and Vimun Sc-3012, the organic solar cells (Fig. 1) and the comparison of these types, a reproducible lighting environment is needed. For that reason a lighting box is build and verified (Fig. 2). Three different lighting concepts are integrated in the reference box which can be regulated using analog dimming. The measurement can be performed using a controllable dc power supply. The three different lighting types are two modes using LEDs for $1200 \mathrm{~lx}$ illuminance (winter in the shade) and $9000 \mathrm{~lx}$ illuminance (summer in the shade) and one mode using halogen lamps for an illuminance of $110000 \mathrm{~lx}$ (summer in the sun). Thereby, first attempts have shown that the high illuminance mode using the halogen lamps is uncritical for all solar cells evaluated regarding the power provided. Following the dc power sup-

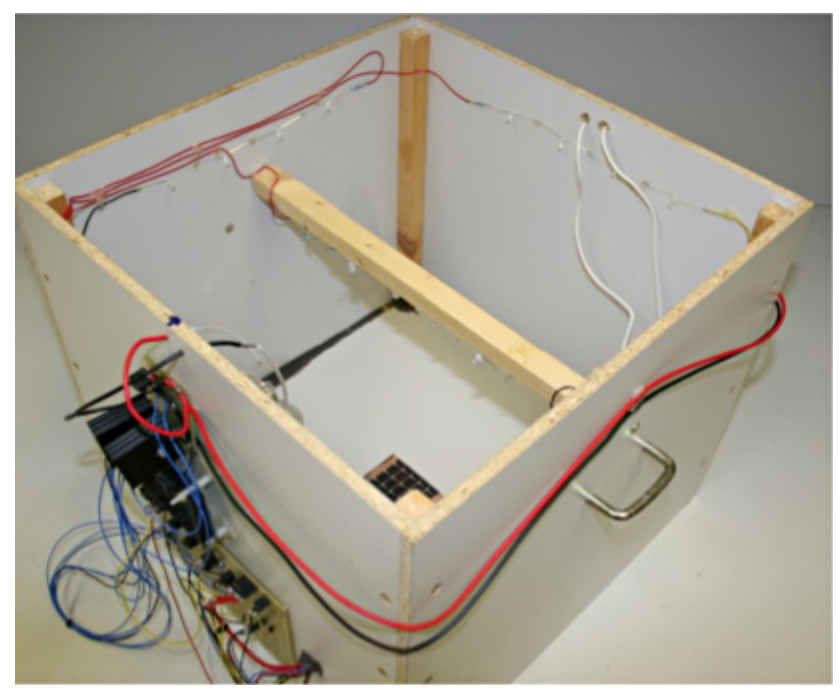

Figure 2. Illuminance box for reference lighting.

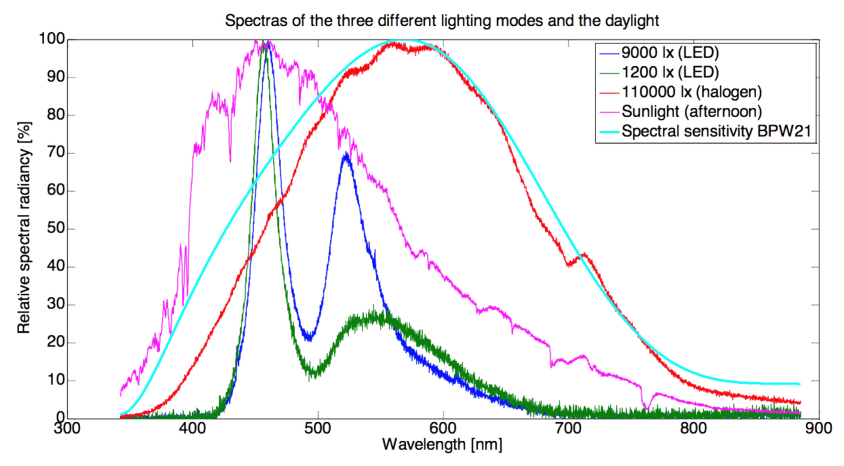

Figure 3. Different spectra of the reference box.

ply for the LED modes starts with the corresponding voltage for 1200 or $9000 \mathrm{~lx}$ illuminance. Then the supply voltage is stepping down until no light is emitting anymore. That implies in case of the $12001 \mathrm{x}$ - or the 9000 lx LED mode that because of the circuitry-wise realization a total number of 280 dimming steps with an increment of $0.01 \mathrm{~V}$ are possible. The illuminance of the different modes is investigated with using a luxmeter with an integrated BPW21 photodiode. This photodiode has approximately the same weighting function as the human eye, so the illuminance measurement in lux is possible. The different spectra of the three different modes, which were recorded using a spectrometer from Aseq, and the spectral sensitivity of the BPW21 photodiode can be seen in Fig. 3.

For a better comparison of the different lighting conditions it would be possible to transform the illuminance into the corresponding irradiance using the photometric radiation equivalent, because the spectrum is known. But for the considered kinds of solar cells there is no need for this transformation, because their spectral sensitivity is approximately equal. 


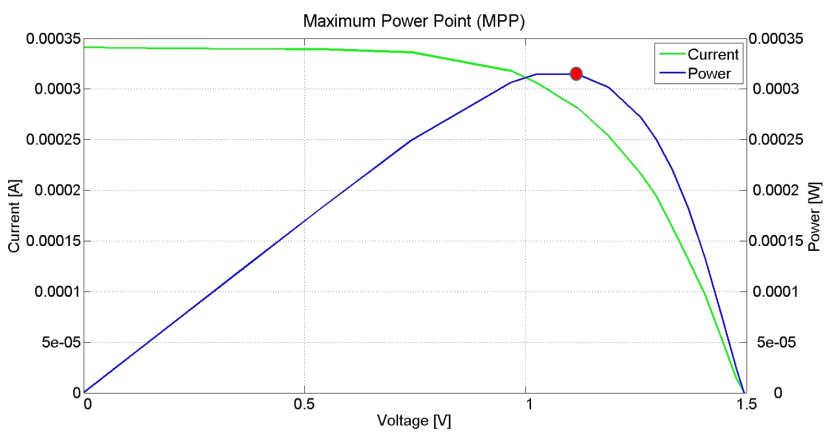

Figure 4. MPP of the Powerfilm SP 3-37 cell with an illuminance of $1200 \mathrm{~lx}$.

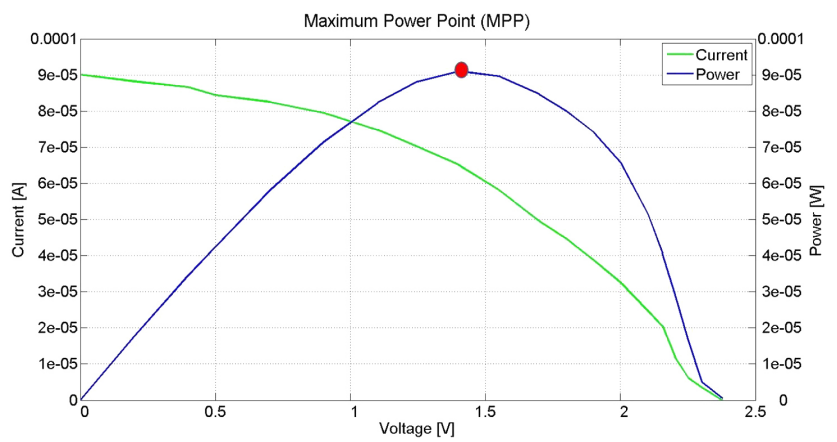

Figure 5. MPP of the organic solar cell with an illuminance $12001 \mathrm{x}$.

To compare the different cells with each other maximum power point (mpp) measurements are used (McEvoy et al., 2011). The maximum power point defines the point of maximum available power in the voltage-current diagram of a solar cell. This point depends on the temperature and the lighting. In this case both, the Vimun Sc-3012 and the organic solar cell are compared under the same illumination conditions at an illuminance of $1200 \mathrm{~lx}$. With less than $1.6 \mathrm{~V}$ maximum voltage (Fig. 4) the Powerfilm SP 3-37 is not guaranteeing the needed voltage at an illuminance of $1200 \mathrm{~lx}$ and is for that reason not suitable for powering the IC. Further on the SP 3-37 would require a lot of space on a possible transponder because of its greater size and is therefore inappropriate for a realization. In Figs. 4-6, the mpp is highlited red.

As shown in Figs. 4-6 such comparatively bad illumination conditions already ensure a power of about $90 \mu \mathrm{W}$. To use the Monza X-2K a voltage of $1.6 \mathrm{~V}$ and a current of $15 \mu \mathrm{A}$ must be guaranteed. Furthermore it is important that the examined organic solar cells are strongly degenerated. That implies that a fully operative organic solar cell can provide five times more power than the evaluated one. The used degenerated organic cell can already ensure a voltage of $1.4 \mathrm{~V}$ and a current of around $65 \mu \mathrm{A}$ in its maximum power point (Fig. 5). If a non-degenerated panel would be used a current of about $300 \mu \mathrm{A}$ would appear at the same voltage.

For the amorphous silicon solar cell Vimun Sc-3012 the mpp is around $0.95 \mathrm{~V}$ at an illuminance of $1200 \mathrm{~lx}$ (Fig. 6).

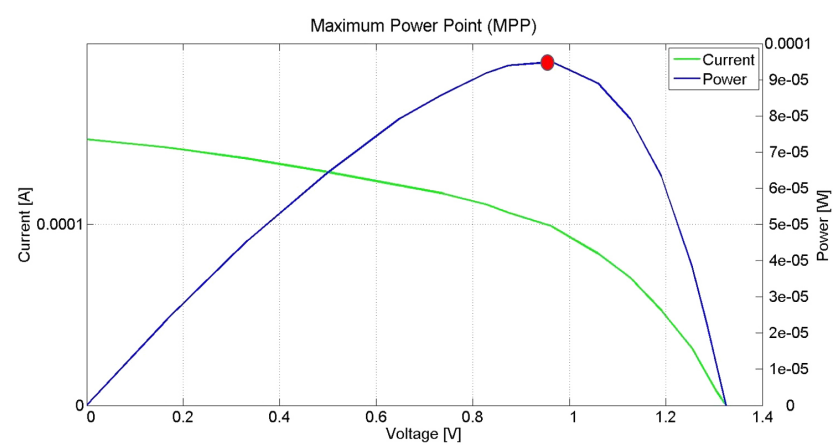

Figure 6. MPP of the Vimun Sc-3012 with an illuminance of $12001 x$.

As a voltage of $1.6 \mathrm{~V}$ is needed to get the IC operational and the maximum voltage is only $1.3 \mathrm{~V}$ a enhanced reading range is not possible. However it is possible to build a series circuit of two Vimun Sc-3012 solar cells. These series connection is ensuring enough current for operating the Monza X-2K consequently. Because of the tiny form factor of the Vimun Sc3012 a second cell can easily be attached at the surface of a possible transponder. The different cells have furthermore been evaluated under higher illuminance, which indicated as uncritical for the service of the RFIC. Further measurement results concerning different types of solar cells are mentioned in Mathews et al. (2014). Thereby GaAs - and dye sensitized solar cells were examined for indoor applications by varying the illuminance.

\section{Used capacitor}

Different capacitors of four electronic contributors got investigated regarding their loading characteristic, leakage current and operating time (Brunelli et al., 2009). To evaluate the loading characteristic of the different capacitors with typical values from 50 to $220 \mathrm{mF}$, it is directly linked to the used solar cells under certain lighting conditions. The time to load a $220 \mathrm{mF}$ capacitor until the minimum operating voltage of the Monza X-2K is reached with respect to an illuminance of $1200 \mathrm{~lx}$ is for the organic non-degraded cell one hour, for the degraded organic cell five hours and for the Vimun Sc-3012 five hours too. If an additionally schottky diode with a typical dropout voltage of $0.2 \mathrm{~V}$ in forward direction is used for discharge protection, the loading time for the non-degraded organic cell increases up to two hours and for the degraded organic cell and the Vimun Sc-3012 up to eight hours assuming an illuminance of $1200 \mathrm{~lx}$. For higher illuminance the required loading time is decreasing very fast. The typical leakage current is around $2 \mu \mathrm{A}$ which is the reason for the voltage drop over time. If a $220 \mathrm{mF}$ capacitor has a voltage of $2.5 \mathrm{~V}$ and exhibits a leakage current of $2 \mu \mathrm{A}$ it takes $35 \mathrm{~h}$ until the voltage drops below $1.6 \mathrm{~V}$. Comparing two different fully loaded capacitors with a capacity of 50 and $220 \mathrm{mF}$ 


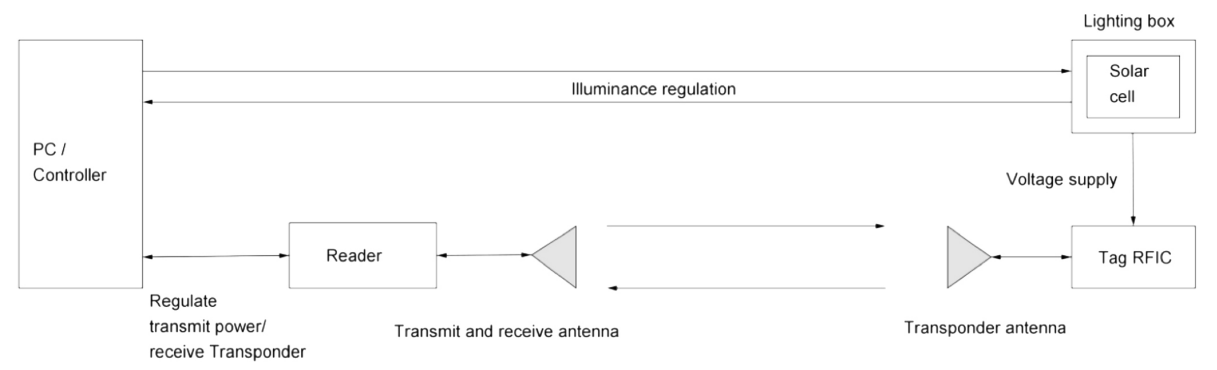

Figure 7. Measurement setup.

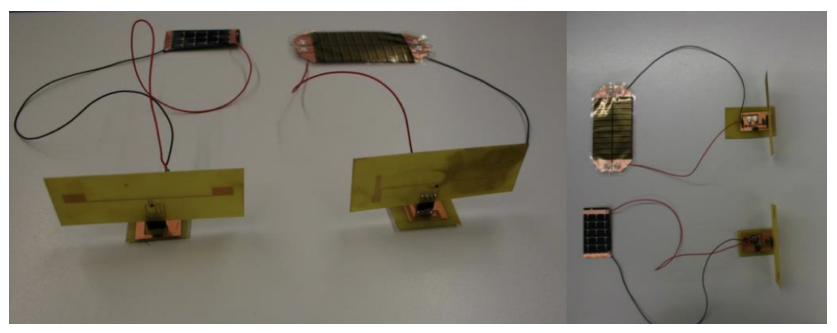

Figure 8. Demonstrators using meander dipole antennas.

concerning their maximum operating time shows, that a capacity of $50 \mathrm{mF}$ allows an operating time of one hour and a $220 \mathrm{mF}$ capacitor an operating time of $7 \mathrm{~h}$. Finally, it appears that the Panasonic Goldcaps are the most common for the intended application. They combine a tiny form factor with a small leakage current and a cheap price.

\section{Range improvement}

Initially different modular demonstrators are build, which allows the evaluation of the possible range improvement using a combination of the examined solar cells, the chosen capacitors and different antenna types. These demonstrators are examined concerning the reachable sensitivity improvement. Thereby, the different solar cells are in a series connection with a schottky diode for discharge protection followed by a supercapacitor in a parallel connection between power and ground. This circuit is connected to the VDD pin of the RFIC. The demonstrators are shown in Fig. 8.

The modularized demonstrators are developed to use different antenna types. One possibility is to use meander dipole antennas, which allows a decrease in required space of the transponder. The effective length remains equal and the directivity is approximately omnidirectional. A second possibility is the usage of planar patch antennas on different substrates. Due to the small bandwidth of about one percent, the high substrate losses using FR4 and the large amount of space needed for a patch antenna lead to the conclusion that this antenna type is impractical for the planned application (Balanis, 2005).

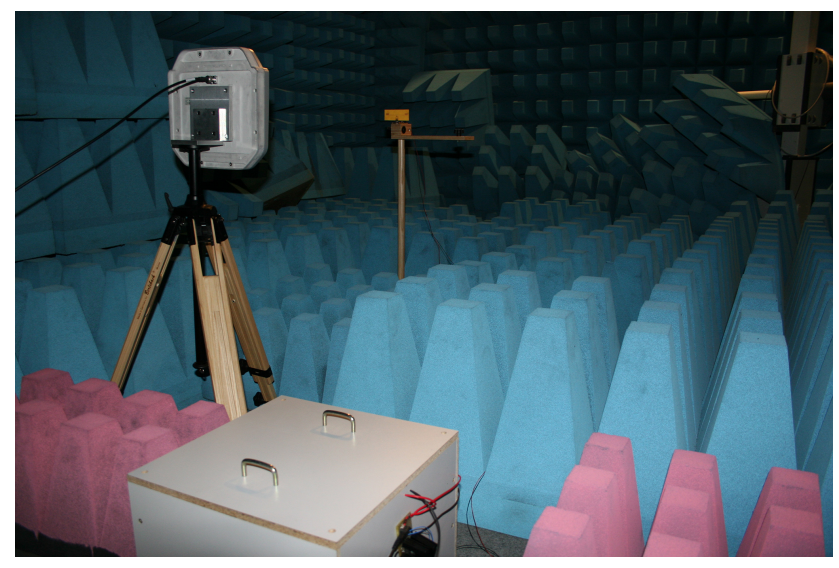

Figure 9. The mentioned setup from Fig. 6 in the anechoic chamber.

The chosen meander dipole antennas can be plugged into a basic module that consists of a capacitor for buffering and a diode as discharge protection. For measurements it is possible to change each of the components, either the antenna, the capacitor or the solar cell.

The read sensitivity depending on the illuminance should be evaluated for the different demonstrator setups. For the following measurements, using the measurement setup shown in Fig. 6, a 3 V reference, the Powerfilm SP 3-37, the Vimun Sc-3012 and the degraded organic cells are compared to each other. The measurements were realized in an anechoic chamber (Fig. 9), with a fixed distance between the reader antenna and the demonstrator of one meter at a height of $91 \mathrm{~cm}$. Two different measurements were made.

First of all the transponder sensitivity for a used dipole antenna with vertical meander structure and a dipole antenna with horizontal meander structure are measured as a function of the used antenna type and the applied solar cell. The illuminance remains constant during one measurement cycle. The read rate (transponder readings per second) was recorded while varying the transmitted power of the reader. It can be seen in Fig. 10 that for the $3 \mathrm{~V}$ reference as well as for the different voltage sources the vertical meander dipole needs $2 \mathrm{~dB}$ less reader sending power then the horizontal dipole. To determine the maximal sensitivity enhancement of the 

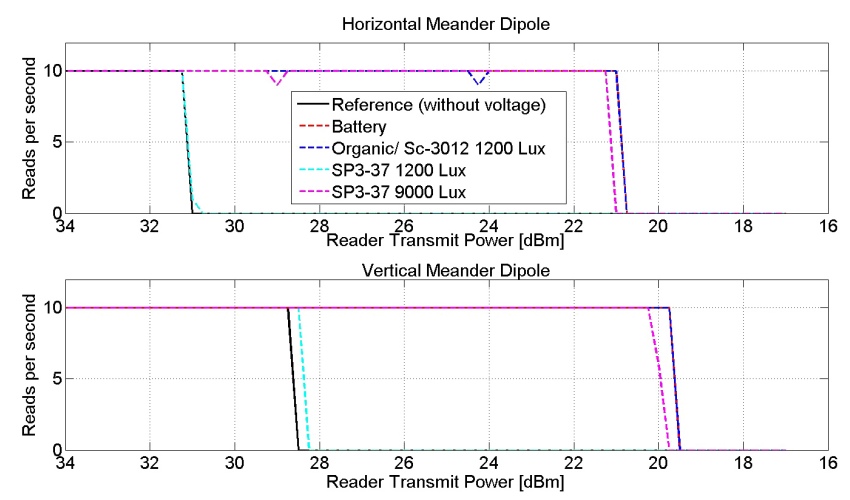

Figure 10. Reads per second using different solar cells and varying the reader sending power.

transponder the minimal necessary reader sending power was detected using a $3 \mathrm{~V}$ reference voltage independent from the illuminance.

Measurements with the Powerfilm SP 3-37, the Vimun Sc3012 and the organic degraded cells at an illuminance of 1200 and $90001 \mathrm{x}$ were done additionally. It showed, that the Vimun Sc-3012 and the organic cells could provide enough power to the RFIC at an illuminance of 1200 lx. The measurement graphs of the Vimun and the organic cells in Fig. 10 are congruent with the $3 \mathrm{~V}$ reference. Furthermore it can be seen, that the additional voltage supply of the RFIC leads to a $9 \mathrm{~dB}$ lower sending power needed at the reader. That $9 \mathrm{~dB}$ less sending power equals a $70 \%$ higher detection range. The listed SP 3-37 did not provide enough supply voltage at $12001 \mathrm{x}$. Even if the illuminance is $9000 \mathrm{~lx}$, there is a narrow difference in the needed reader sending power compared to the $3 \mathrm{~V}$ reference.

A second performance evaluation is realized to examine the necessary illuminance to guarantee the maximal sensitivity improvement of the transponder. For this evaluation only the vertical meander dipole antenna is used as the same result can be seen for the horizontal meander dipole.

The measurements in Fig. 11 have shown, that the sensitivity improvement for the organic solar cells and a series circuit of two Vimun Sc-3012 start at different illuminances. With an illuminance of $287 \mathrm{~lx}$ the sensitivity improvement initiates for the organic solar cells and reaches its maximum at a value of $3511 \mathrm{x}$. This effect appears considerably later for the series circuit of the two Vimun cells. With an illuminance of $491 \mathrm{~lx}$ the sensitivity improvement initiates and reaches its maximum value at $748 \mathrm{~lx}$.

Regarding the mentioned measurement setup and the performed evaluations it is possible to decide whether the solar cells of an energy harvesting transponder are suitable for the envisaged application. In summary, the organic solar cells are more suitably at bad lighting conditions. However, the organic cells require more space than the conventional amor-
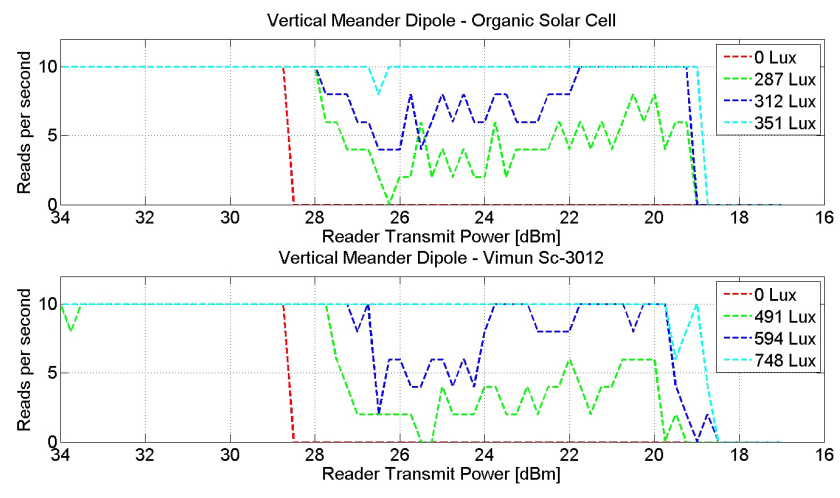

Figure 11. Sensitivity improvement depending on the illuminance for the organic solar cell and the Vimun Sc-3012 using a vertical meander dipole antenna.

phous silicon solar cells and are more expensive disposed of their development stage.

\section{Conclusions}

Organic solar cells and amorphous silicon solar cells are proposed and evaluated to power RFICs that contain a pin for an external voltage supply in order to increase the read range of a transponder under bad lighting conditions. The cells are evaluated by maximum power point measurements using a reproducible measurement environment. To guarantee the higher sensitivity of potential semi-active transponder, even if the cells are darkened for a short period of time, an adequate capacitor is chosen. Finally a modular demonstrator is build with the possibility to use different antenna types. This demonstrator is used to examine the transponder sensitivity improvement depending on the illuminance regarding the different types of solar cells. As a result of the examinations a $70 \%$ higher detection range could be ensured using organic cells starting at an illuminance of 351 and 748 lx in case of the Vimun silicon solar cells. With the measurement setup shown in Fig. 7 it is possible to make a decision whether the solar cells of an energy harvesting transponder are suitable for the desired application. Future work consists of developing a completely integrated transponder using organic solar cells for indoor applications.

Acknowledgements. The authors acknowledge the Fraunhofer ISE for providing the organic solar cells used in this work.

Edited by: J. Anders

Reviewed by: two anonymous referees 


\section{References}

Balanis, C. A.: Antenna Theory, 3rd Edn., John Wiley \& Sons, Hoboken, USA, 2005.

Brunelli, D., Moser, C., Thiele, L., and Benini, L.: Design of a Solar-Harvesting Circuit for Batteryless Embedded Systems, IEEE T. Circ. Syst., 1, 2519-2528, 2009.

Dennler, G. and Sariciftci, N. S.: Flexible Conjugated PolymerBased Plastic Solar Cells: From Basics to Applications, Proc. IEEE, 93, 1429-1439, 2005.

Finkenzeller, K.: RFID Handbuch, 5. Aktualisierte und erweiterte Auflage, Carl Hanser Verlag GmbH, Munich,, 2008.

Georgiadis, A. and Collado, A.: Improving Range of Passive RFID Tag Utitilizing Energy Harvesting and High Efficiency Class-E Oscillators, Centre Tecnologic de Tele-comunications de Catalunya (CTTC), Prague, 2011.

Glunz, S. W., Dicker, J., Esterle, M., Hermle, M., Isenberg, J., Kamerewerd, F. J., Knobloch, J., Kray, D., Leimenstoll, A., Lutz, F., Osswald, D., Preu, R., Rein, S., Schaffer, E., Schetter, C., Schmidhuber, H., Schmidt, H., Steuder, M., Vorgrimler, C., and Willeke, G.: High-Efficiency Silicon Solar Cells for LowIllumination Applications, IEEE Photovoltaic Specialist Conference (PVSC), New Orleans, USA, 2002.
Mathews, I., Kelly, G., King, P. J., and Frizzell, R.: GaAs solar cells for Indoor Light Harvesting, 40th IEEE Photovoltaic Specialist Conference (PVSC), Denver, USA, 2014.

McEvoy, A., Markvart, T., and Castaner, L.: Practical Handbook of Photovoltaics: Fundamentals and Applications, 2nd Edn., Academic Press, Waltham, 2011.

Sample, A. P., Braun, J., Parks, A., and Smith, J. R.: Photovoltaic Enhanced UHF RFID Tag Antennas for Dual Purpose Energy Harvesting, IEEE International Conference on RFID 2011, Orlando, USA, 2011.

Spies, P., Pollak, M., and Rohmer, G.: Energy Harvesting for Mobile Communication Devices, 29. Int. Telecommunications Energy Conference, Fraunhofer ILS, Nürnberg, 2007. 